\title{
Imaging of spontaneous neuromagnetic activity in a patient with internal carotid artery stenosis
}

Sir,

Magnetoencephalography (MEG) is beginning to 
be applied clinically because this technique allows direct capture of cerebral neural activity and resolves the problem associated with low spatial resolution of electroencephalography (EEG). The present study measured spontaneous cerebral magnetic fields using a whole-head-type MEG system, and used frequency analysis to attempt imaging of cerebral ischemic areas.

A 76-year-old man suffered a ischemic attack resulting in left hemiparesis, and brain magnetic resonance imaging (MRI) confirmed a small infarct in the watershed area of the right parieto-occipital region [Figure 1a]. Cerebral angiography confirmed approximately $80 \%$ stenosis at the origin of the right internal carotid artery [Figure 1b]. Positron emission tomography by the ${ }^{15} \mathrm{O}$ gas inhalation (steady-state) method $\left({ }^{15} \mathrm{O}\right.$ gas PET) confirmed a decrease in cerebral blood flow $(\mathrm{CBF})$ in the region of the right middle cerebral artery (right $36.2 \mathrm{ml} / 100 \mathrm{~g} / \mathrm{min}$; left $40.7 \mathrm{ml} / 100 \mathrm{~g} / \mathrm{min}$ ) [Figure 2a] and increased oxygen extraction fraction (OEF) (right 45.2\%; left 37.7\%) [Figure 2b]. The patient was thus diagnosed to have reduced CBF and metabolism reserve (misery perfusion) in the right middle cerebral artery region. Using a 160-channel whole-head-type gradiometer (MEG vision PQ1160C; Yokogawa, Kanazawa, Japan), ${ }^{[1]}$ MEG was performed to measure spontaneous cerebral neuromagnetic activities. Slow-wave component analysis was performed using an adaptive beamformer, which provided a kind of spatial filtration. ${ }^{[2]}$ At a sampling frequency of $500 \mathrm{~Hz}$, a
DC-200 Hz bandpass filter and a 50-Hz notch filter were used; 150 -s data were analyzed in terms of $\delta$ waves (0.3$4 \mathrm{~Hz}$ ) and $\theta$ waves $(4-8 \mathrm{~Hz})$. Asymmetrical slow-wave distributions in the cerebral cortex within $3 \mathrm{~cm}$ of the brain surface were superimposed onto preoperative cerebral MRI scans. MEG showed that the distribution of $\delta$ waves was broad from the right frontal area to the parietal area in the ipsilateral cerebral hemisphere corresponding to ischemic areas as confirmed by PET [Figure 3a], while the distribution of $\theta$ waves was relatively localized from the posterior temporal area to the parietal area in the ipsilateral cerebral hemisphere [Figure 3b].

Studies on slow-wave distributions as assessed by MEG have been previously conducted ${ }^{[3,4]}$ These studies surmised that $\delta$ wave distributions are seen around the cerebral infarction, while $\theta$ waves are in a relatively localized area irrespective of the extent of ischemia. However, in the past, slow-wave analysis was performed by comparing raw data or estimating equivalent current dipoles (ECDs), and such techniques are not suited to estimating ischemic areas beyond a certain volume in the brain, and imaging has not been necessarily clear. We addressed this problem by using the adaptive beamformer method, which allows reconstruction of cerebral activity sources with high spatial resolution without limits to numbers. ${ }^{[2]}$

The results of the present study suggest that cerebral

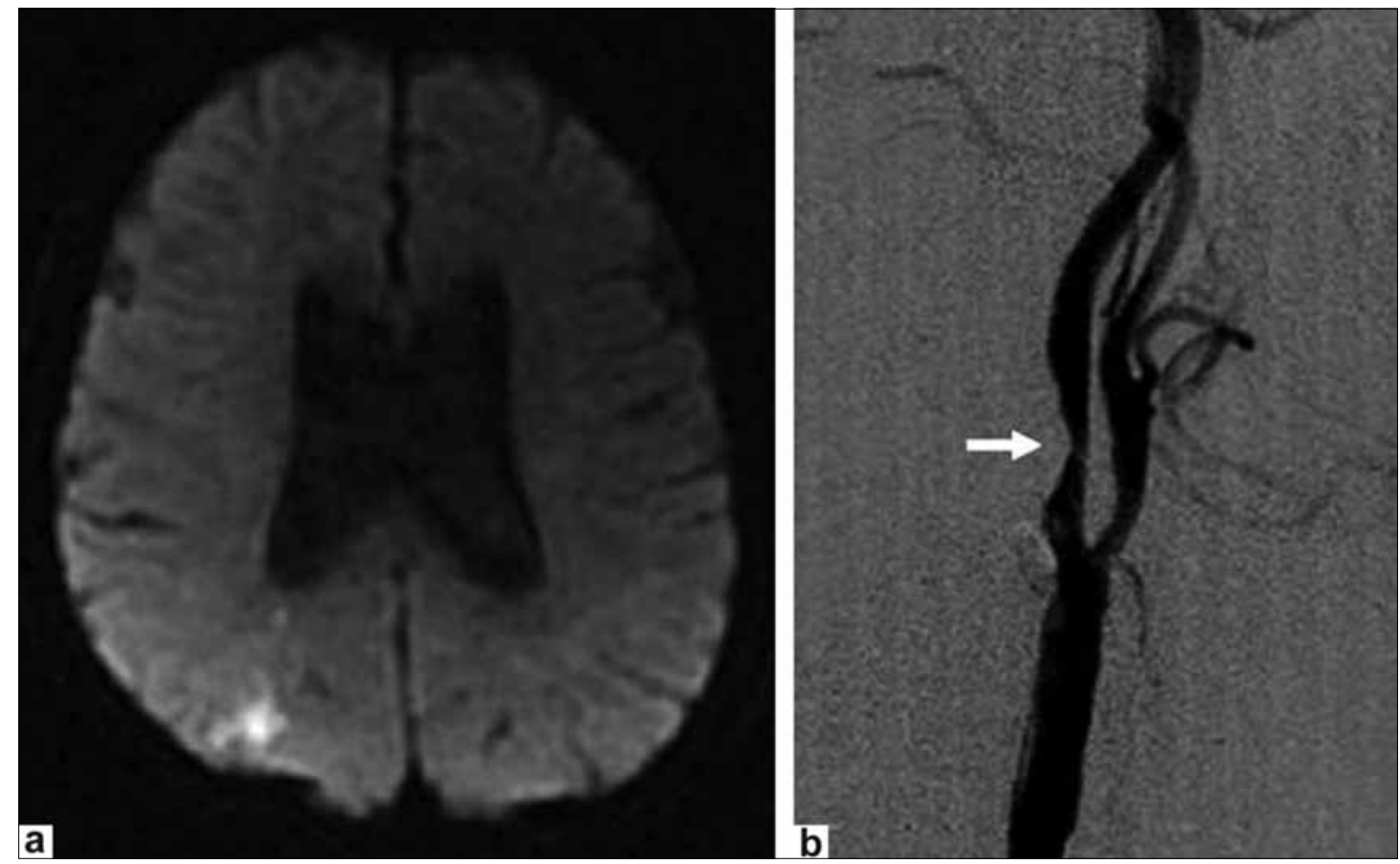

Figure 1: (a) Diffusion-weighted MRI at onset of transient ischemic attack. Small infarct lesion is seen in watershed area of right parieto-occipital region; (b) Cerebral angiography. Approximately $\mathbf{8 0 \%}$ stenosis is seen at origin of right internal carotid artery (arrow) 

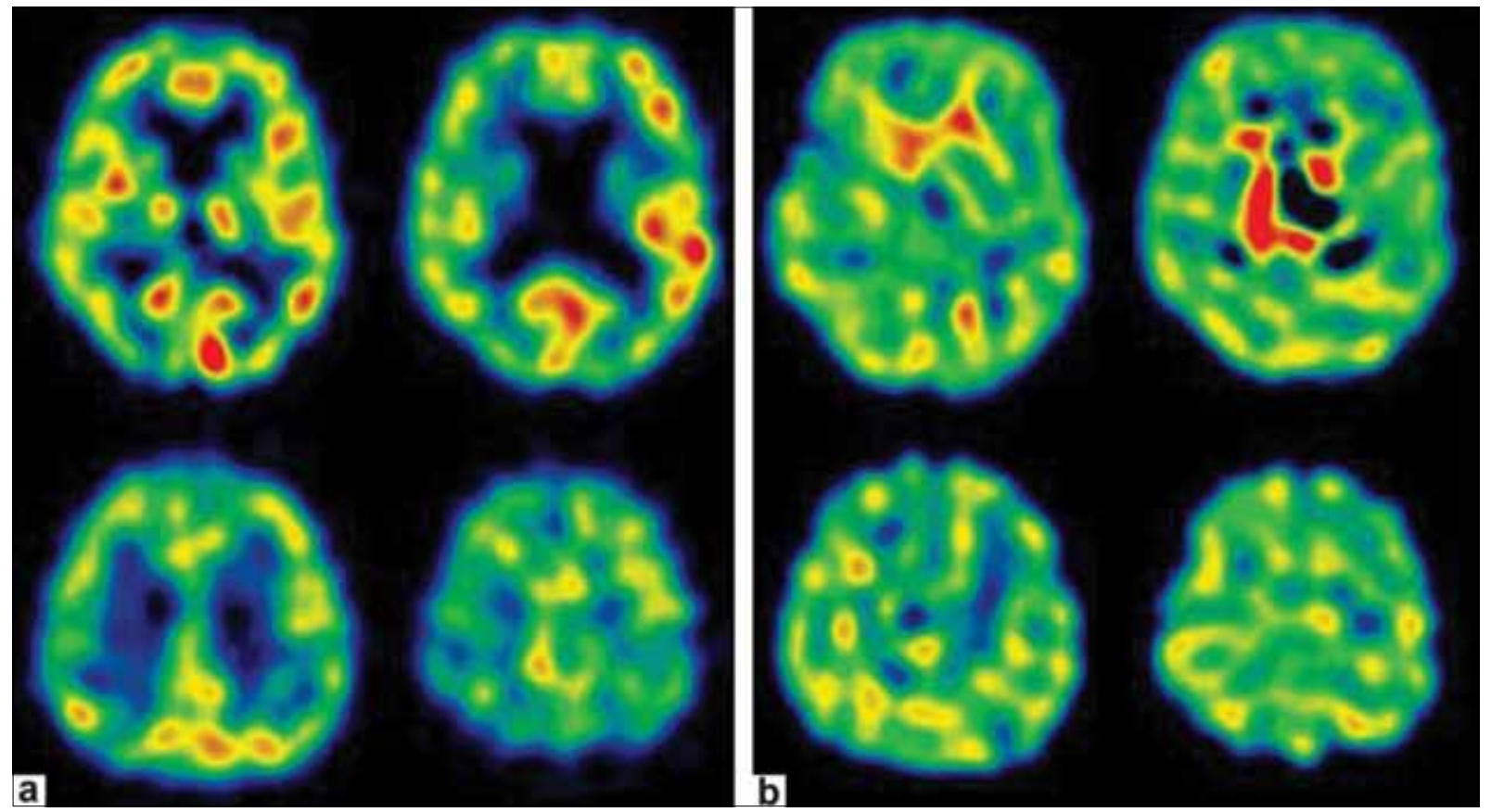

Figure 2: (a) Preoperative ${ }^{15} \mathrm{O}$-gas PET (CBF). Reduced cerebral blood flow is apparent in cortex of right cerebral hemisphere (right: $36.2 \mathrm{ml} / 100 \mathrm{~g} / \mathrm{min}$; left: $40.7 \mathrm{ml} / 100 \mathrm{~g} / \mathrm{min}$ ); (b) Preoperative ${ }^{15} \mathrm{O}-$ gas PET (OEF). Increased oxygen extraction fraction is seen in cortex of right cerebral hemisphere (right: $45.2 \%$; left: $37.7 \%$ )
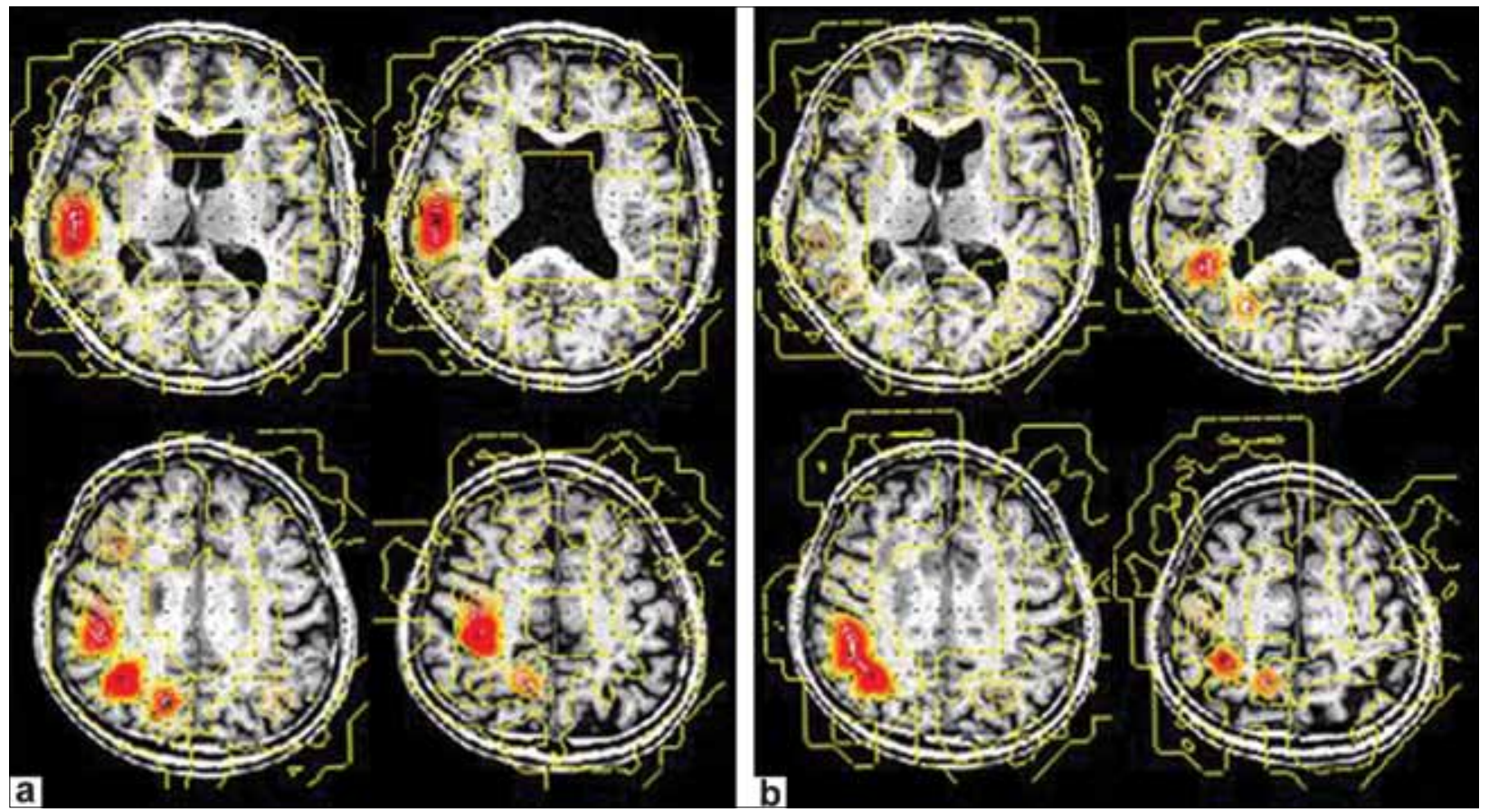

Figure 3: (a) MEG slow-wave analysis $(\delta 0.3-4 \mathrm{~Hz})$. Increased slow-wave intensities are seen over broad area in right temporal, frontal and parietal lobes; (b) MEG slow-wave analysis $(\theta$ 4-8 Hz). Slow-wave intensities are increased over relatively narrow area from right posterior temporal area to parietal area

magnetic field frequency analysis using MEG has the potential to identify the area of cerebral ischemia and may represent a useful technique for visualizing the ischemic penumbra. ${ }^{[5]}$ Future investigations of differences between $\delta$ and $\theta$ wave distributions will be necessary in greater numbers of patients.
Shinichi Sakamoto ${ }^{1,2}$, Naohiro Tsuyuguchi ${ }^{2}$, Wataru Ide ${ }^{1}$, Ikuo Hashimoto ${ }^{1}$, Hajime Kamada ${ }^{1}$, Hiroaki Tanaka ${ }^{3}$, Kensuke Sekihara ${ }^{4}$, Kenji Ohata ${ }^{2}$

${ }^{1}$ Departments of Neurosurgery, Hokuto Hospital, Obihiro, ${ }^{2}$ Osaka City University Graduate School of Medicine, Osaka, 
${ }^{3}$ MEG Center, Yokogawa Electric Corporation, Kanazawa,

${ }^{4}$ Systems Design and Engineering,

Tokyo Metropolitan University, Tokyo, Japan.

E-mail: s-sakamoto@med.osaka-cu.ac.jp

DOI: 10.4103/0028-3886.55584

\section{References}

1. Higuchi M, Shimogawara M, Haruta Y, Uehara G, Kawai J, Ogata H, et al. System integration and trade-offs of SQUID system for biomagnetic applications. Appl Superconduct 1998;5:441-9.

2. Sekihara K, Nagarajan SS, Poeppel D, Marantz A, Miyashita Y. Reconstructing spatio-temporal activities of neural sources using an MEG vector beamformer technique. IEEE Trans Biomed Eng 2001;48:760-71.

3. Kamada K, Saguer M, Möller M, Wicklow K, Katenhäuser M, Kober H, et al. Functional and metabolic analysis of cerebral ischemia using magnetoencephalography and proton magnetic resonance spectroscopy. Ann Neurol 1997;42:554-63.

4. Seki S, Nakasato N, Ohtomo S, Kanno A, Shimizu H, Tominaga T. Neuromagnetic measurement of unilateral temporo-parietal theta rhythm in patients with internal carotid artery occlusive disease. Neuroimage 2005;25:502-10.

5. Astrup J, Siesjö BK, Symon L. Thresholds in cerebral ischemia - The ischemic penumbra. Stroke 1981;12:723-5.

Accepted on 09-01-2009 